\title{
A COMPARISON OF LIDAR REFLECTANCE AND RADIOMETRICALLY CALIBRATED HYPERSPECTRAL IMAGERY
}

\author{
A. Roncat ${ }^{\mathrm{a}, *}$, C. Briese $^{\mathrm{a}, \mathrm{b}}$, N. Pfeifer ${ }^{\mathrm{a}}$ \\ ${ }^{\text {a }}$ Research Groups Photogrammetry and Remote Sensing, Department of Geodesy and Geoinformation, TU Wien, Vienna, Austria \\ (andreas.roncat, christian.briese, norbert.pfeifer)@geo.tuwien.ac.at, www.geo.tuwien.ac.at \\ ${ }^{\mathrm{b}}$ EODC Earth Observation Data Centre for Water Resources Monitoring GmbH, Vienna, Austria \\ christian.briese@eodc.eu. Www.eodc.eu
}

Commission VII, WG VII/6

KEY WORDS: Radiometry, Calibration, Laser Scanning, Hyperspectral

\begin{abstract}
:
In order to retrieve results comparable under different flight parameters and among different flight campaigns, passive remote sensing data such as hyperspectral imagery need to undergo a radiometric calibration. While this calibration, aiming at the derivation of physically meaningful surface attributes such as a reflectance value, is quite cumbersome for passively sensed data and relies on a number of external parameters, the situation is by far less complicated for active remote sensing techniques such as lidar.

This fact motivates the investigation of the suitability of full-waveform lidar as a "single-wavelength reflectometer" to support radiometric calibration of hyperspectral imagery. In this paper, this suitability was investigated by means of an airborne hyperspectral imagery campaign and an airborne lidar campaign recorded over the same area. Criteria are given to assess diffuse reflectance behaviour; the distribution of reflectance derived by the two techniques were found comparable in four test areas where these criteria were met. This is a promising result especially in the context of current developments of multi-spectral lidar systems.
\end{abstract}

\section{INTRODUCTION}

Commercial full-waveform lidar systems have become ever increasingly available and used for the production of high-resolution 3D topographic information in the past decade. Full-waveform lidar additionally inherits the possibility of assigning physically meaningful target attributes, e.g. the backscatter cross-section or a diffuse-reflectance value, to the resulting $3 \mathrm{D}$ point cloud in the same spatial resolution. This allows for the derivation of quantities comparable for different sensors, emitted laser energies, other flight-campaign parameters and acquisition dates.

While the process of radiometric calibration for lidar data as an active remote sensing technique is rather straightforward (Wagner, 2010 Briese et al., 2012), for passively sensed optical data such as hyperspectral imagery (HSI), several additional variables have to be considered. Concerning the airborne case, these variables are typically given in resolutions much coarser than the ground sampling distance of the optical sensor.

Airborne lidar, also known as airborne laser scanning (ALS), has already proven to be a valuable geometric source for facilitating radiometric calibration of hyperspectral imagery (Schneider et al., 2014). Beyond that, the aforementioned lidar-derived surface attributes, especially diffuse reflectance, may also serve as a radiometric input for calibrating hyperspectral data in an according wavelength domain, acting as a "single-wavelength reflectometer" in an area-wide sense. In this study, we present a method for validating the lidar-derived diffuse surface reflectance and and its comparison to according values calculated from HSI data. The hypothesis is evaluated by means of an extended fullwaveform lidar campaign and an HSI campaign, both recorded over the Lägern area, located northwest of Zurich, Switzerland.

The paper is organized as follows: The underlying physical and mathematical frameworks for radiometric calibration of both li-

\footnotetext{
${ }^{*}$ Corresponding author.
}

dar and passively sensed data are presented in Section 2 followed by the method for comparing the reflectances retrieved by each of this techniques in Section 3 The subsequent section describes the used data sets. Results are given in Section 5 , conclusions are drawn in the last section.

\section{THEORY}

In this section, we will present the physical-mathematical framework for radiometric calibration for both lidar and passively sensed optical data.

\subsection{Radiometric Calibration of Lidar Data}

The basic relation of the transmitted power of a laser pulse $P_{t}(t)$ and the recorded echo power $P_{e}(t)$ is given by the radar equation (Jelalian, 1992 Wagner, 2010):

$$
P_{e}(t)=\frac{D_{r}^{2}}{4 \pi R^{4} \beta_{t}^{2}} P_{t}\left(t-\frac{2 R}{v_{g}}\right) \sigma \eta_{\mathrm{SYS}} \eta_{\mathrm{ATM}},
$$

with $\beta_{t}$ being the beamwidth of the transmitted signal, $R$ denoting the distance from the sensor to the target, $t$ the travel time, $v_{g}$ the group velocity of the laser ray (approx. speed of light in vacuum), $D_{r}$ the receiving aperture diameter, $\eta_{\mathrm{SYS}}$ the system transmission factor and $\eta_{\mathrm{ATM}}$ the atmospheric transmission factor. The scattering cross-section $\sigma$ (in $\mathrm{m}^{2}$ ) summarizes target characteristics:

$$
\sigma:=\frac{4 \pi}{\Omega_{S}} \rho A_{L} \cos \vartheta
$$

The term $A_{L}\left[\mathrm{~m}^{2}\right]$ is the area of the laser footprint, i.e. the area formed by the intersection of the cone and a sphere with centre at the laser's position and radius $R$ corresponding to the distance from the laser to the target, while $\Omega_{S}$ [sr] is the solid angle of the scattering cone. The unitless quantity $\rho$ is the reflectivity of the target surface in scattering direction. The angle $\vartheta$ is the incidence 
angle, i.e. the angle formed by the direction of the laser beam and the local surface normal of the target surface; see Figure 1 .

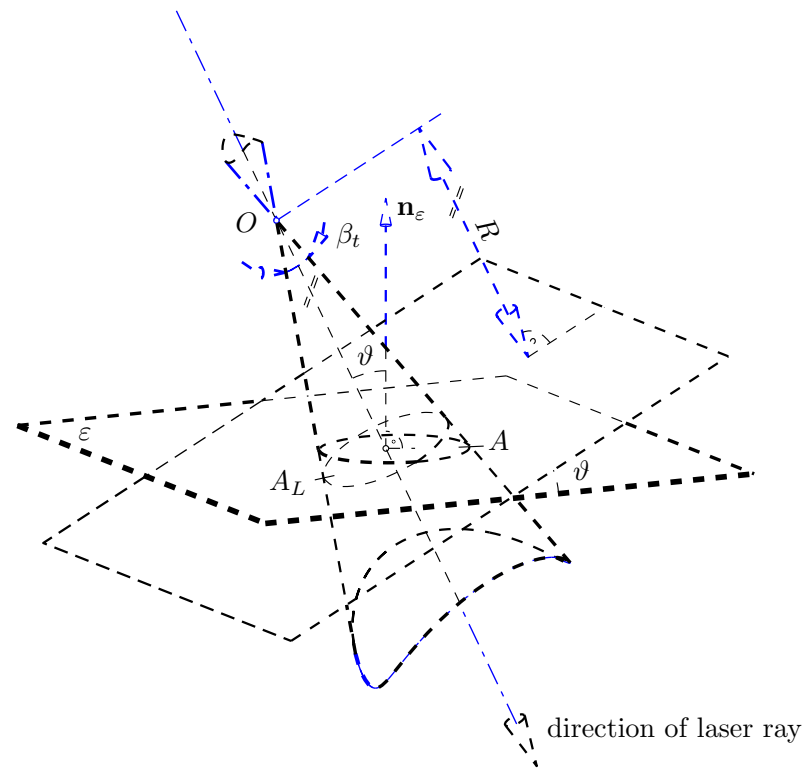

Figure 1: Geometric parameters of the radar equation. The angle $\vartheta$ formed by the laser ray and the normal of of the target plane $\varepsilon$ is called incidence angle (Roncat et al., 2012).

In a monostatic configuration, i.e. the transmitter and receiver being close together, $\sigma$ is to be considered as backscatter crosssection. This is the case for practically all airborne lidar systems. If the target surface exhibits a diffuse reflectance behaviour, the scattering solid angle $\Omega_{S}=\pi$ and the diffuse surface reflectance $\rho_{d}$ is derived as

$$
\rho_{d}=\frac{\sigma}{4 A_{L} \cos \vartheta} .
$$

The retrieval of $\sigma$ and/or $\rho_{d}$ is known as radiometric calibration. For this purpose, unknown but constant quantities are summarized as calibration constant, determined by means of reference targets which might be surfaces of (assumed) known and constant reflectance (Wagner et al., 2006), natural or artificial surfaces with calibrated reflectance behaviour (Lehner and Briese, 2010, Kaasalainen et al., 2009).

If the last factor in Equation (1), $\eta_{\mathrm{ATM}}$, can be assumed as constant throughout the flight campaign, it is included in the calibration constant and not determined independently. If such an assumption does not hold, $\eta_{\text {ATM }}$ can be formulated as a function of the range $R$ and of an (constant) atmospheric attenuation coefficient $a[\mathrm{~dB} / \mathrm{km}]$ (Höfle and Pfeifer, 2007):

$$
\eta_{\mathrm{ATM}}(R)=10^{-\frac{2 R a}{10000}}
$$

or, in the case of a more complex atmospheric situation, be calculated per target w.r.t. to atmospheric parameters, flight altitude and terrain elevation by applying look-up tables as e.g. implemented in the ATCOR software (Richter and Schläpfer, 2016). An assessment of the variation of $\eta_{\mathrm{ATM}}$ for the dataset investigated in this study is given in Section 4

The actual formulation of the calibration constant and sequence of calibration steps may vary w.r.t. (a) which input data is available, e.g. an intensity value per point, amplitude and echo width derived by full-waveform data, additional amplitude and width of the transmitted signal, or a deconvolved echo waveform. Further: (b) which quantities can be assumed as constants and (c) which quantity is sought as result of radiometric calibration (Briese et al., 2012 Roncat, 2014). Additionally to $\sigma$ and $\rho_{d}$, also a normalized backscatter cross-section $\sigma^{0}$ and a backscatter coefficient $\gamma$ (both unitless) may be the quantity of interest in radiometric calibration.

While the backscatter cross-section $\sigma$ can be derived from data only related to the single 3D point of interest itself, the diffuse reflectance requires knowledge of the spatial neighbourhood of this point, expressed e.g. by the local surface-normal vector, commonly derived using the $3 \mathrm{D}$ point cloud of the same lidar campaign.

\subsection{Radiometric Calibration of passively sensed optical Data}

In addition to the aforementioned factors to be considered in radiometric calibration, passively sensed data are also dependent on various other quantities. In a first step, a gain factor and an offset have to be applied, transferring the digitized pixel values to at-sensor radiances $L_{\lambda}$, given in $W /\left(\mathrm{m}^{2} \mathrm{sr} \mu \mathrm{m}\right)$ or equivalent. To derive further the surface reflectance $\rho$, the following parameters are to be considered (Moran et al., 1992, Chander et al., 2009):

- the mean solar exo-atmospheric irradiance,

- the solar zenith angle,

- the Earth-Sun distance,

- the path radiance,

- the atmospheric transmittance in viewing and illumination direction, resp., and

- the downwelling diffuse irradiance.

Their values are commonly given in models of coarser resolution than the one of imagery data.

\section{METHOD}

In order to support HSI radiometric calibration by lidar-derived reflectance, areas are to be determined where a diffuse surface reflectance can be assumed, i.e. where the reflectance is independent of the viewing direction of the sensor. This is an important prerequisite as the surfaces are likely to be viewed from different positions in a lidar and an HSI campaign, resp.

As a first criterion, the lidar-derived reflectance is only valid for extended targets, i.e. the target area exceeding the the one of the laser beam footprint. Therefore, only single echoes per laser shot can be taken into consideration. Additionally, the echo ratio is to be considered. This quantity is the number of points in the 3D neighbourhood (i.e. a sphere) of a (grid) point, $n_{3 \mathrm{D}}$, divided by the number of points $n_{2 \mathrm{D}}$ in the 2D neighbourhood of this point, i.e. a cylinder of the same radius (Höfle et al., 2009):

$$
\operatorname{ER}[\%]=\frac{n_{3 \mathrm{D}}}{n_{2 \mathrm{D}}} \cdot 100,
$$

with $n_{3 \mathrm{D}} \leq n_{2 \mathrm{D}}$. The echo ratio can be considered as an opacity measure in vertical direction. See Figure 2 for explanatory examples.

The assumption of diffuse reflectance can further be validated by comparing the derived values among neighbouring flight strips in their area of overlap (Lehner and Briese, 2010 Roncat et al., 2014) as these areas are viewed from different directions if we 


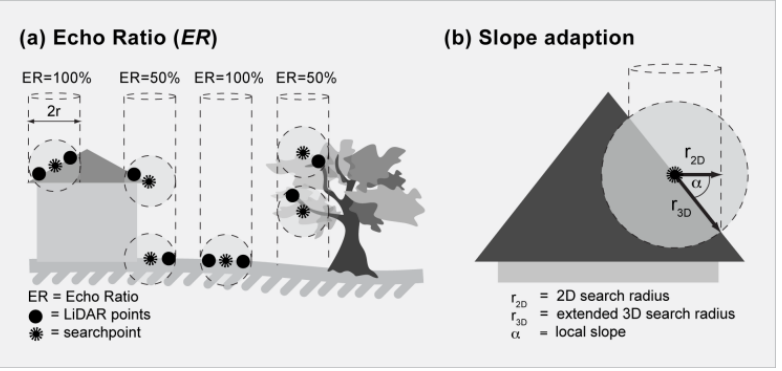

Figure 2: Definition of the Echo Ratio ER (Höfle et al., 2009).

compare one flight strip to the other. In case a certain threshold of the absolute value of their difference is exceeded, the hypothesis of diffuse reflectance is to be rejected. Moreover, the assumption of a well-defined normal vector of the target surface can be evaluated using the standard error of unit weight $\sigma_{0}$ of the normal vector calculation. This $\sigma_{0}$ can be considered as a measure of surface roughness. Only where all three criteria are met:

(a) extended and opaque targets,

(b) isotropic reflectance behaviour, and

(c) a well-defined surface-normal vector,

the reflectance behaviour of the surface may be considered as diffuse.

\section{DATA SETS}

The data investigated in this study were recorded over the Lägern area, a wooded mountainous area in the Jura mountains, located northwest of Zurich, Switzerland. The lidar campaign was carried out on August 1, 2010 using a RIEGL LMS-Q680 full-waveform instrument, operating at a wavelength of $1,550 \mathrm{~nm}$ (Riegl LMS, 2016). The point density was approx. 50 points per $\mathrm{m}^{2}$; The campaign consisted of 15 flight strips and covered an area of about $12.5 \mathrm{~km}^{2}$.

Hyperspectral imagery data were recorded over the Lägern area on July 18, 2014 using an APEX sensor (Hueni et al., 2009); the wavelengths ranged from $400 \mathrm{~nm}-2,500 \mathrm{~nm}$. The wavelength of the band used for comparison to the lidar data was $1,551 \mathrm{~nm}$ $($ FWHM $=22 \mathrm{~nm})$. The spatial resolution of the HSI data was about $1.5 \mathrm{~m}$ at ground; reflectance values were resampled to a $2 \mathrm{~m}$ grid in the Swiss National Coordinate System CH1903. The overlapping area of the two campaigns is mostly forested but contains grasslands, fields and built-up areas as well. Based on the criteria for assessing diffuse surface reflectance in lidar data presented in the previous section, four test areas were chosen, illustrated by red rectangles in Figure 4 The respective reflectance values were resampled to a $2 \mathrm{~m}$ grid. A description of the test areas in detail (see also Figure 3):

Test area 1: area of homogeneous reflectance behaviour. The distribution of the reflectance in both datasets, expressed by the median and the $\sigma_{M A D}$, was assumed to be uni-modal and comparable.

Test areas 2 and 3: areas covered with surface types of different reflectance, i.e. roads, grassland and fields. In addition to the distribution, the linear relationship between the two datasets, expressed by the coefficient of determination $R^{2}$, was investigated as well.
Test area 4: a more complex scene in a built-up area where the dependence of median and $\sigma_{M A D}$ on a minimum echo ratio was assessed.

An overview of the whole campaigns' setup is shown in Figure 4
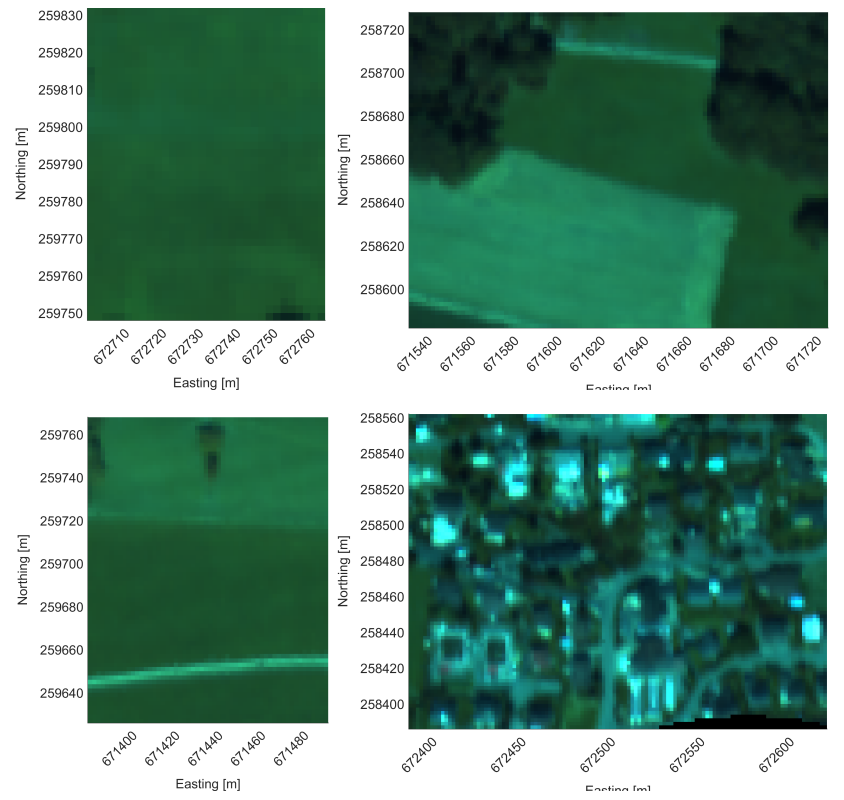

Figure 3: The four test areas in detail, shown as RGB true-color composite of the according HSI reflectances. Top left: test area 1, bottom left: test area 2 . Top right: test area 3, bottom right: test area 4.

The radiometric calibration of the lidar dataset and the calculation of normal vectors on a per-point level as well as the interpolation of rasters for reflectance, echo ratio and strip differences were performed using the lidar software suite OPALS (OPALS, 2016), developed by TU Wien. The HSI reflectance grid was delivered by University of Zurich.

Concerning the variation of the atmospheric transmission coefficient $\eta_{\text {ATM }}$ within the lidar dataset, the maximum relative differences to a mean value of $\eta_{\text {ATM }}$ were assessed, applying (a) an attenuation coefficient of $a=0.22 \mathrm{~dB} / \mathrm{km}$ and (b) atmospheric look-up tables, resp. In both cases, the relative variations did not exceed $\pm 2 \%$. Therefore, $\eta_{\text {ATM }}$ was regarded as constant for this dataset.

\section{RESULTS}

The four test areas showed that the variations in the lidar-derived reflectances, expressed by $\sigma_{M A D}$, were slightly higher than the ones in the HSI-derived reflectances. This might be due to the higher spatial resolution of the original raw lidar data and the resulting capability of assessing reflectance variations in higher detail. The numerical results are given in Table 1

Test areas 2 and 3 showed $R^{2}$ values of 0.53 and 0.59 for the lidar- and HSI-derived reflectance, resp. In both test areas, a slight increase in $R^{2}$ for increasing minimum echo ratio up to $95 \%$ was discovered. Test area 4 exhibited a noticeable decrease in both median and $\sigma_{M A D}$ of the lidar reflectances for increasing minimum echo ratio; this effect was less prominent in the HSI reflectances. In all cases, the median values were found comparable for lidar and HSI reflectance data. See Figures 5 to 8 for a graphical representation of the results in the four test areas. 


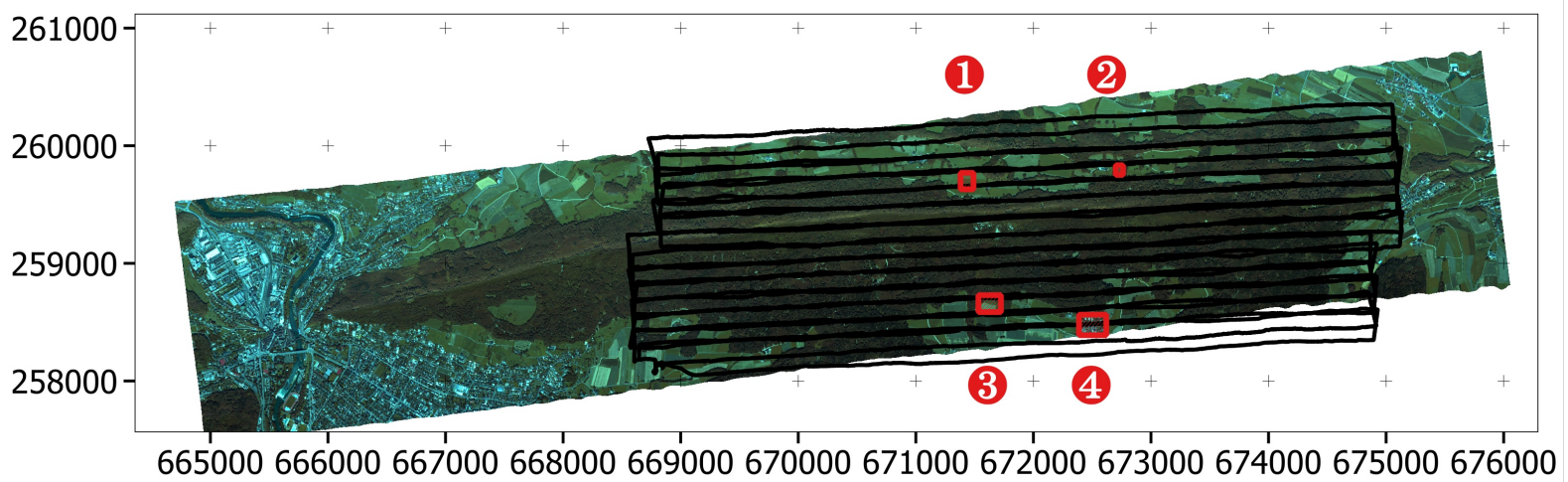

Figure 4: Overview of the lidar and the HSI campaign recorded over the Lägern area. An RGB composite of the according HSI reflectances is shown in the background. The outlines of the lidar strips are shown in black and the four test areas are depicted by red rectangles. Coordinate system: Swiss National Grid CH1903.

\begin{tabular}{ccccc} 
& lidar & & HSI & \\
test area & median & $\sigma_{M A D}$ & median & $\sigma_{\text {MAD }}$ \\
\hline 1 & 0.184 & 0.017 & 0.221 & 0.020 \\
2 & 0.164 & 0.049 & 0.180 & 0.030 \\
3 & 0.219 & 0.133 & 0.215 & 0.110 \\
4 & 0.172 & 0.087 & 0.176 & 0.059
\end{tabular}

Table 1: Reflectance distributions for the four test areas, evaluated for a minimum echo ratio of $75 \%$ each. Detailed results w.r.t. varying minimum echo ratio for test area 4 are given in Table 2
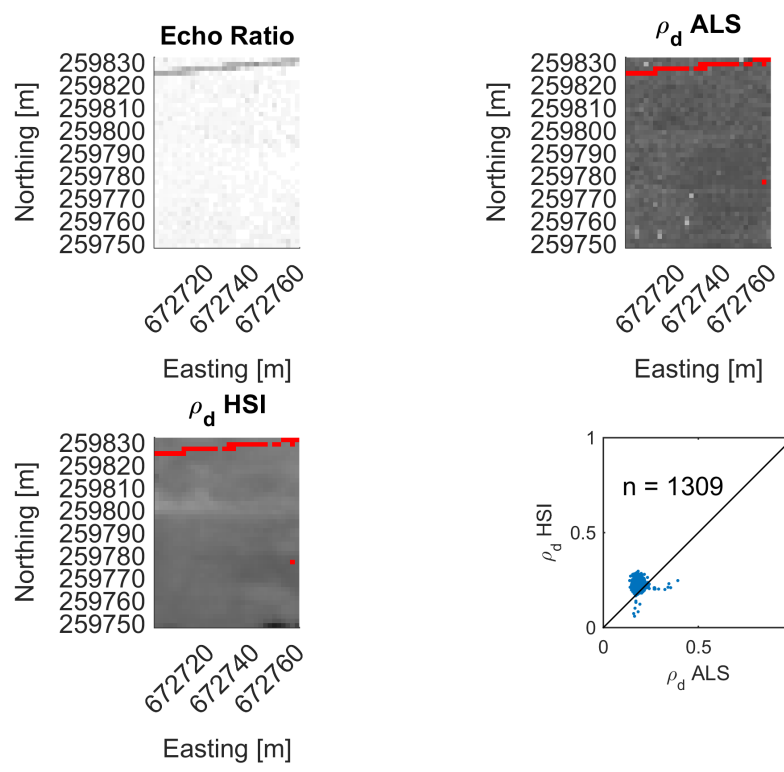

Figure 5: Results for test area 1, evaluated for a minimum echo ratio of $75 \%$. Reflectance values are shown in grayscale from 0 (black) to 1 (white) and the echo ratio is visualized analogously. Pixels excluded from the analysis due to an echo ratio below the threshold are shown in red.

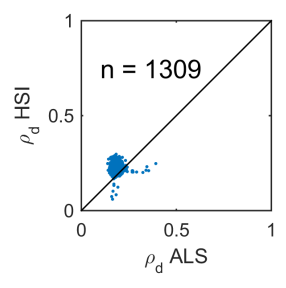

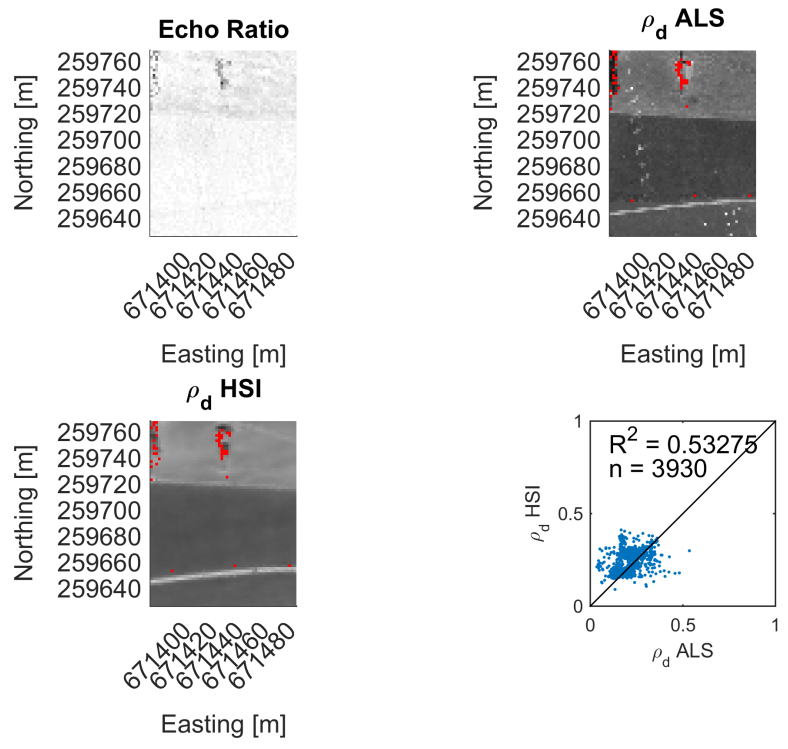

Figure 6: Results for test area 2, evaluated for a minimum echo ratio of $75 \%$. Reflectance values are shown in grayscale from 0 (black) to 1 (white) and the echo ratio is visualized analogously. Pixels excluded from the analysis due to an echo ratio below the threshold are shown in red. See also Table 2

\begin{tabular}{cccccc} 
& \multicolumn{3}{c}{ lidar } & \multicolumn{3}{c}{ HSI } \\
min. ER & \# pixels & median & $\sigma_{M A D}$ & median & $\sigma_{M A D}$ \\
\hline $50 \%$ & 9709 & 0.174 & 0.089 & 0.176 & 0.059 \\
$55 \%$ & 9364 & 0.173 & 0.088 & 0.176 & 0.059 \\
$60 \%$ & 8880 & 0.174 & 0.089 & 0.176 & 0.059 \\
$65 \%$ & 8212 & 0.173 & 0.088 & 0.176 & 0.059 \\
$70 \%$ & 7398 & 0.172 & 0.087 & 0.176 & 0.059 \\
$75 \%$ & 6524 & 0.170 & 0.087 & 0.177 & 0.060 \\
$80 \%$ & 5701 & 0.167 & 0.086 & 0.178 & 0.060 \\
$85 \%$ & 4910 & 0.161 & 0.083 & 0.179 & 0.061 \\
$90 \%$ & 4169 & 0.155 & 0.080 & 0.179 & 0.062 \\
$95 \%$ & 3366 & 0.140 & 0.070 & 0.178 & 0.061 \\
$100 \%$ & 2457 & 0.128 & 0.065 & 0.174 & 0.057
\end{tabular}

Table 2: Reflectance distribution in test area 4 w.r.t. to a minimum echo ratio (ER). See also Figure 8 

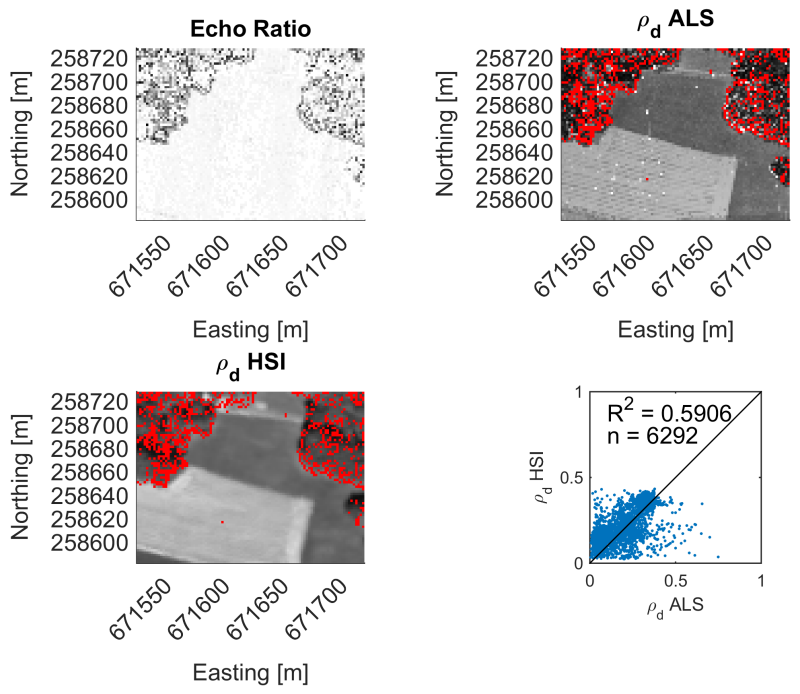

Figure 7: Results for test area 3, evaluated for a minimum echo ratio of $75 \%$. Reflectance values are shown in grayscale from 0 (black) to 1 (white) and the echo ratio is visualized analogously. Pixels excluded from the analysis due to an echo ratio below the threshold are shown in red.

\section{DISCUSSION AND OUTLOOK}

This study dealt with the radiometric comparison of full-waveform lidar and passively sensed hyperspectral data. Lidar, as an active remote sensing technique, needs by far less assumptions for performing radiometric calibration. This has led to the motivation for testing lidar as a "single-wavelength reflectometer" in an area-wide sense in order to support radiometric calibration of hyperspectral imagery in an according wavelength.

Criteria for the validity of the diffuse surface reflectance, assessable in an advanced lidar-data processing, were formulated and a comparison of reflectance data was performed in four test areas within an extended airborne HSI and lidar campaign, resp.

The distribution of the reflectance, expressed by the median and the $\sigma_{M A D}$ values, was found comparable in all four test areas, giving empirical evidence for the validity of the hypothesis. While this study concentrated on a single wavelength, current developments of multi-spectral lidar systems (Briese et al., 2012. Hakala et al., 2012 Wallace et al., 2014) give the motivation for further research in this field.

\section{ACKNOWLEDGEMENTS}

The research leading to these results has received funding from the European Union's 7th Framework Programme (FP7/2014 2018) under EUFAR2 contract no. 312609.

The Lägern ALS dataset was processed at RSL (UZH) (Schneider et al., 2014).

The Lägern APEX imaging reflectometer dataset has been acquired within the Swiss Earth Observatory Network (SEON) project and was processed using the APEX Processing and Archiving Facility (Hueni et al., 2009).

\section{REFERENCES}

Briese, C., Pfennigbauer, M., Lehner, H., Ullrich, A., Wagner, W. and Pfeifer, N., 2012. Radiometric calibration of multiwavelength airborne laser scanning data. In: ISPRS Annals of
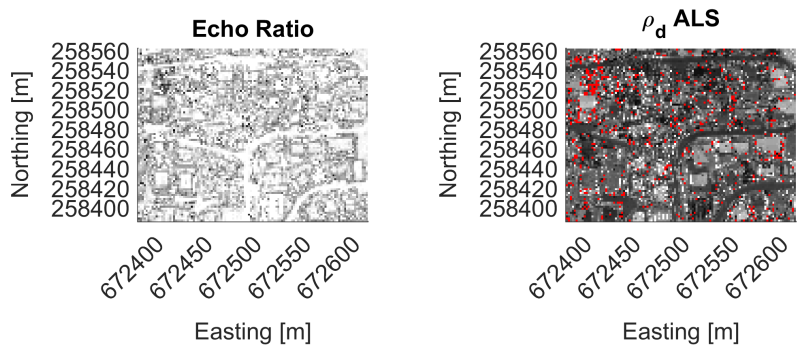

$\rho_{\mathrm{d}} \mathbf{H S I}$
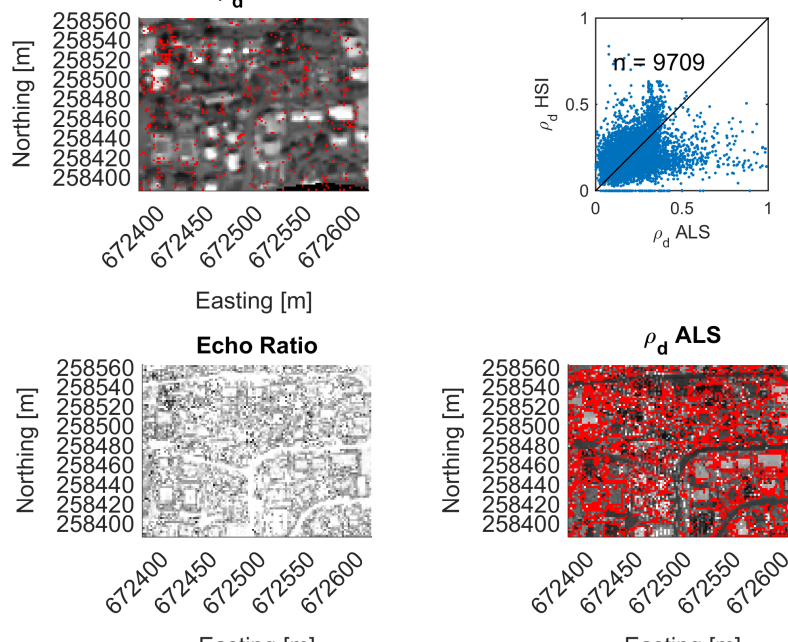

Easting $[\mathrm{m}]$

$\rho_{\mathbf{d}} \mathbf{H S I}$
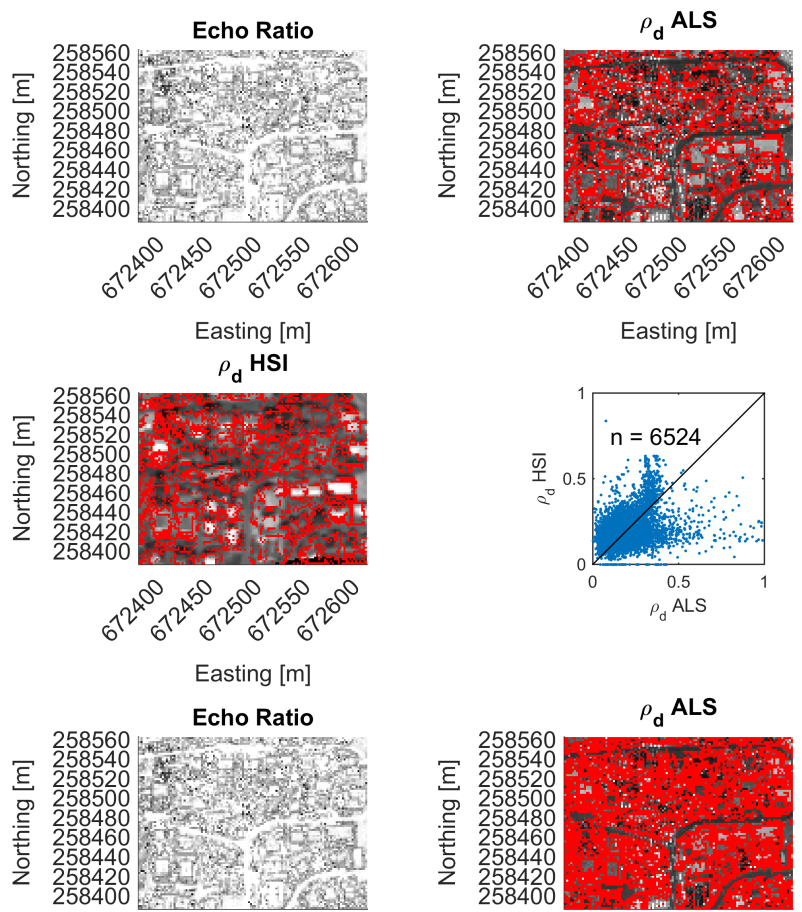

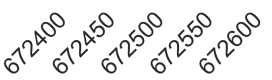

Easting [m]

$\rho_{\mathbf{d}} \mathrm{HSI}$

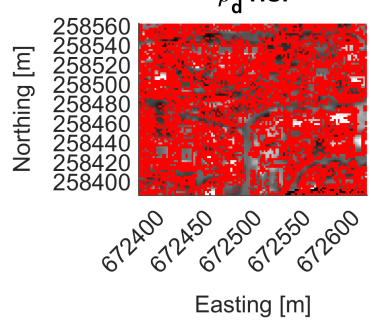

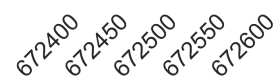

Easting $[\mathrm{m}]$

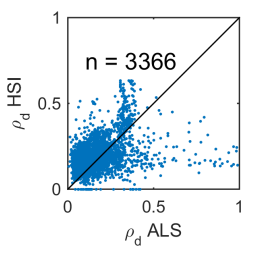

Figure 8: Results for test area 4, evaluated for a minimum echo ratio of $50 \%$ (top), $75 \%$ (middle) and $95 \%$ (bottom). Reflectance values are shown in grayscale from 0 (black) to 1 (white) and the echo ratio analogously. Pixels excluded from the analysis due to an echo ratio below the respective threshold are shown in red. 
the Photogrammetry, Remote Sensing and Spatial Information Sciences 1 (Part 7), Melbourne, Australia, pp. 335-340. ISSN: 1682-1750.

Chander, G., Markham, B. L. and Helder, D. L., 2009. Summary of current radiometric calibration coefficients for landsat MSS, TM, ETM+, and EO-1 ALI sensors. Remote Sensing of Environment 113(5), pp. 893-903.

Hakala, T., Suomalainen, J., Kaasalainen, S. and Chen, Y., 2012. Full waveform hyperspectral lidar for terrestrial laser scanning. Optics Express 20(7), pp. 7119-7127.

Höfle, B. and Pfeifer, N., 2007. Correction of laser scanning intensity data: Data and model-driven approaches. ISPRS Journal of Photogrammetry and Remote Sensing 62(6), pp. 415-433.

Höfle, B., Mücke, W., Dutter, M., Rutzinger, M. and Dorninger, P., 2009. Detection of building regions using airborne LiDAR A new combination of raster and point cloud based GIS methods. In: Proceedings of the Geoinformatics Forum Salzburg, Salzburg, Austria, pp. 66-75.

Hueni, A., Biesemans, J., Meuleman, K., Dell'Endice, F., Schläpfer, D., Adriaensen, S., Kempenaers, S., Odermatt, D., Kneubuehler, M., Nieke, J. and Itten, K., 2009. Structure, components and interfaces of the airborne prism experiment (APEX) processing and archiving facility. IEEE Transactions in Geosciences and Remote Sensing 47(1), pp. 29-43.

Jelalian, A. V., 1992. Laser Radar Systems. Artech House, Boston.

Kaasalainen, S., Hyyppä, H., Kukko, A., Litkey, P., Ahokas, E., Hyyppä, J., Lehner, H., Jaakkola, A., Suomalainen, J., Akujarvi, A., Kaasalainen, M. and Pyysalo, U., 2009. Radiometric calibration of lidar intensity with commercially available reference targets. IEEE Transactions on Geoscience and Remote Sensing 47(2), pp. 588-598.

Lehner, H. and Briese, C., 2010. Radiometric calibration of full-waveform airborne laser scanning data based on natural surfaces. In: ISPRS Technical Commission VII Symposium 2010: 100 Years ISPRS - Advancing Remote Sensing Science. International Archives of the Photogrammetry, Remote Sensing and Spatial Information Sciences 38 (Part 7B), Vienna, Austria, pp. 360365.

Moran, M. S., Jackson, R. D., Slater, P. N. and Teillet, P. M., 1992. Evaluation of simplified procedures for retrieval of land surface reflectance factors from satellite sensor output. Remote Sensing of Environment 41(2), pp. 169-184.

OPALS, 2016. Orientation and Processing of Airborne Laser Scanning data (product homepage). Department of Geodesy and Geoinformation - Research Groups Photogrammetry and Remote Sensing. http://geo.tuwien.ac.at/opals (16 Apr. 2016).

Richter, R. and Schläpfer, D., 2016. Atmospheric/Topographic Correction for Airborne Imagery. ReSe Applications, Wil SG, Switzerland. ATCOR-4 User Guide, Version 7.0.3, March 2016 (16 Apr. 2016).

Riegl LMS, 2016. Homepage of the company RIEGL Laser Measurement Systems GmbH. http://www.riegl.com. (16 Apr. 2016).

Roncat, A., 2014. Backscatter Signal Analysis of Small-Footprint Full-Waveform Lidar Data. PhD thesis. Supervisors: Norbert Pfeifer (TU Vienna), Uwe Stilla (TU Munich).

Roncat, A., Morsdorf, F., Briese, C., Wagner, W. and Pfeifer, N., 2014. Laser Pulse Interaction with Forest Canopy: Geometric and Radiometric Issues. Managing Forest Ecosystems, Vol. 27, Springer Netherlands, Dordrecht, The Netherlands, chapter 2, pp. $19-41$.
Roncat, A., Pfeifer, N. and Briese, C., 2012. A linear approach for radiometric calibration of full-waveform Lidar data. In: Proc. SPIE 8537, Image and Signal Processing for Remote Sensing XVIII.

Schneider, F. D., Leiterer, R., Morsdorf, F., Gastellu-Etchegorry, J.-P., Lauret, N., Pfeifer, N. and Schaepman, M. E., 2014. Simulating imaging spectrometer data: 3D forest modeling based on LiDAR and in situ data. Remote Sensing of Environment 152, pp. 235-250.

Wagner, W., 2010. Radiometric calibration of small-footprint full-waveform airborne laser scanner measurements: Basic physical concepts. ISPRS Journal of Photogrammetry and Remote Sensing 65(6, ISPRS Centenary Celebration Issue), pp. 505-513. Wagner, W., Ullrich, A., Ducic, V., Melzer, T. and Studnicka, N., 2006. Gaussian decomposition and calibration of a novel smallfootprint full-waveform digitising airborne laser scanner. ISPRS Journal of Photogrammetry and Remote Sensing 60(2), pp. 100112.

Wallace, A., McCarthy, A., Nichol, C., Ren, X., Morak, S., Martinez-Ramirez, D., Woodhouse, I. and Buller, G., 2014. Design and evaluation of multispectral LiDAR for the recovery of arboreal parameters. IEEE Transactions on Geoscience and Remote Sensing 52(8), pp. 4942-4954. 